International Journal of Clinical \& Medical Allergy (IJCMA)

ISSN 2332-2799

\title{
Anaphylactic Reaction Induced By Paclitaxel in the Treatment of Non-Small Cell Lung Cancer (NSCLC)
}

A. Tiotiu ${ }^{1 *}$, C. Clément-Duchene ${ }^{1}$, P. Vaillant ${ }^{1}$, Y. Martinet ${ }^{1}$

19 Rue du Morvan, CHU Brabois Nancy, Service de Pneumologie, Bat. Spécialités Médicales, 54511 Vandoeuvreles-Nancy, France.

\section{Abstract}

Most systemic chemotherapic agents used in lung cancer treatment can lead to hypersensitivity reactions, of varying degrees of severity. In absence of laboratory tests (such as elevated serum total tryptase or/and plasma histamine levels).Distinguishing between anaphylaxis and acute infusion reactions is impossible since their symptoms are identical.

Few Paclitaxel hypersensitivity reactions described in literature are documented in the absence of standardized tests to chemotherapeutics drugs and since not all suspected patients undergo allergological tests. A 74 years old female patient, with history of Inobitriol induced laryngeal angioedema and right middle lobe NSCLC, developed a moderate anaphylactic reaction during Paclitaxel second administration. Infusion suspension and symptomatic treatments led to rapid symptoms remission. Prick tests to Paclitaxel were negatives but the intradermal reaction was positive, as well as basophile activation test.

The mechanism of paclitaxel-associated hypersensitivity reaction is uncertain and its solvent vehicle may be involved; in case of paclitaxel immediate hypersensitivity reaction, we recommend tryptase and/or histamine serum levels quantification to confirm the acute anaphylaxis episode and realization of allergologicals tests to Paclitaxel and its solvent vehicle (skin tests \pm challenge test) 3 to 4 weeks after the incident. Rapid desensitization is possible for both Ig-E mediated and non-Ig-E mediated immediate hypersensitivity reactions to Paclitaxel.

Keywords: Paclitaxel Hypersensitivity; Non-Small Cell Lung Cancer; Allergological Tests; Desensitization

\section{*Corresponding Author:}

A. Tiotiu,

9 Rue du Morvan, CHU Brabois Nancy,

Service de Pneumologie, Bat. Spécialités Médicales,

54511 Vandoeuvreles-Nancy, France.

Tel: +33383153580; Fax: +33383153541

E-mail: atiotiu@yahoo.com

Received: August 25, 2013

Accepted: September 07, 2013

Published: September 10, 2013

Citation: A. Tiotiu,C. Clément-Duchene, P. Vaillant, Y. Martinet (2013) Anaphylactic Reaction Induced By Paclitaxel in the Treatment of NonSmall Cell Lung Cancer (NSCLC). Int J Clin Med Allergy. 1(1), 1-2.doi: http://dx.doi.org/10.19070/2332-2799-130001

Copyright: A. Tiotiu $^{\circ}$ 2013. This is an open-access article distributed under the terms of the Creative Commons Attribution License, which permits unrestricted use, distribution and reproduction in any medium, provided the original author and source are credited.

\section{Introduction}

The diagnosis of anaphylaxis is based on clinical criteria, hypotension and shock being not mandatory. Anaphylaxis is unpredictable, underrecognized by patients and underdiagnosed by professionals. [1]

Most chemotherapic agents can lead to hypersensitivity reactions, and it is impossible to distinguish between anaphylaxis and acute infusion reactions since their symptoms are identical. Paclitaxel hypersensitivity reactions incidence is high ( $8 \%-45 \%)$ but with standard premedication (corticosteroids and antihistamines) it drops to $<10 \%[2]$.
We describe a case of documented anaphylactic reaction to paclitaxel and suggest practical advices for improving its diagnostic strategy.

\section{Case report}

A 74 years old female was treated with Carboplatin $(375 \mathrm{mg}$ ) and Paclitaxel $(299 \mathrm{mg}$ ) (once every three weeks) after surgery for a right middle lobe NSCLC (p'T2N2) and before radiotherapy. Despite premedication with dexamethasone, dexchlorpheniramine, ranitidine, five minutes after the second Paclitaxel infusion start, she presented an anaphylactic reaction with dyspnea, bronchospasma, tachycardia, nausea, vomiting, generalized erythema and itching. Hemodynamic and respiratory parameters were preserved. After infusion cessation, a rapid remission was obtained with oxygen, antihistamines, systemic corticosteroids, intravenous antiemetic and inhaled bronchodilators. Paclitaxel was subsequently replaced by Vinorelbine with a good tolerance.

One month before surgery she did experienced a laryngeal angioedema, 5 minutes after intravenous injection of iodinated contrast agents Iobitridol; prick, intradermal and patch tests for these agents were negative and Iodixanol was well tolerated.

Nine weeks after anaphylactic reaction, prick tests to Paclitaxel $(0.01 \mathrm{mg} / \mathrm{ml}, 0.1 \mathrm{mg} / \mathrm{ml}, 1 \mathrm{mg} / \mathrm{ml})$ were negative. Intradermal reaction was positive at $0.01 \mathrm{mg} / \mathrm{ml}(9 \mathrm{~mm}$ to $18 \mathrm{~mm}$ papule increase with erythema). Basophile activation test to Paclitaxel was positive.

\section{Comments}

Paclitaxel hypersensitivity reactions are frequently described in lit- 
erature but few are documented since not all suspected patients undergo allergological tests and in the absence of standardized allergological tests to chemotherapeutics drugs.

The mechanism of Paclitaxel-associated hypersensitivity reaction is uncertain, it may be non-immunological but due to direct histamine release by mast cells. Because of water insolubility, $\mathrm{Pa}-$ clitaxel is used with a solvent system Cremophor EL, that can cause hypersensitivity reactions, neurotoxicity, and alter Paclitaxel pharmacokinetics. [2] The respective roles of Paclitaxel and Cremophor EL are not well defined, since Cremophor EL can cause anaphylactic reactions via complement activation.[3]

Our patient symptoms were moderate and it was impossible to determine if the reaction was anaphylactic or acute infusion reaction.[2] Because our patient had two risk factors for anaphylaxis: age [1] and a laryngeal angioedema episode induced by Iobitridol [4], we suspected Paclitaxel as trigger of anaphylaxis. Skin tests and basophile activation test confirmed Paclitaxel as cause of the anaphylactic reaction.

Recently, a case of IgE-mediated reaction to Paclitaxel was proved by skin and in vitro testing [5]. We didn't have the opportunity to perform an IgE dot-blot assay for our patient.

For patients with immediate hypersensitivity reactions to Paclitaxel, we recommend the quantification of serum tryptase (increased 15-180 minutes after symptom onset) and/or plasma histamine levels (increased 15-60 minutes after symptom onset) to confirm anaphylaxis [1] and realization of allergological tests to Paclitaxel and Cremophor EL (skin tests \pm challenge test) 3-4 weeks after the incident. Both Ig-E mediated and non-Ig-E mediated immediate hypersensitivity reactions of any severity are amenable to rapid desensitization. [5]

\section{References}

[1]. Simons FE. Anaphylaxis: Recent advances in assessment and treatment. J Allergy Clin Immunol 2009;124: 625-636

[2]. Lenz HJ. Management and preparedness for infusion and hypersensitivity reactions. The Oncologist 2007;12: 601-609

[3]. Szebeni J. Complement activation-related pseudoallergy: a new class of drug-induced acute immune toxicity. Toxicology 2005; 216: 106-121

[4]. Castells MC, Tennant NM, Sloane DE, Hsu FI, Barrett NA and al. Hypersensitivity reactions to chemotherapy: outcomes and safety of rapid desensitization in 413 cases. J Allergy Clin Immunol 2008; 122: 574-580.

[5]. Prieto GarciaA, Pineda de la Losa F.Immunoglobulin E-mediated severe anaphylaxis to Paclitaxel. J Investig Allergol Clin Immunol 2010; 20: 170-171 